\title{
Efficiency and consistency in group decisions*
}

\author{
TAPAN BISWAS \\ Department of Economics, University of Hull, Hull, HU6 7RX, UK
}

Accepted 23 October 1992

\begin{abstract}
The paper introduces a concept of "efficiency set" in the context of group decisions and analyses its properties. If the set contains a single element, then the Borda rule finds it. Otherwise, the group needs a value function to choose from the efficient alternatives. Two value functions, with considerations for the number of participants who are badly affected by the choice, have been discussed. It turns out that the consistency axiom of group choice imposes a constraint, on the form of the value function, with questionable normative significance.
\end{abstract}

\section{Introduction}

The possibility or impossibility of the construction of a complete social ordering of actions or events from individual preferences is one of the core issues in the literature on public choice theory. It is also recognized that the theory of public choice is more concerned with the choice set than with a complete social ordering (Sen, 1970). If the social choice set does not exist, then some kind of voting procedure becomes necessary. One of the simple voting procedures suggested in the literature is known as Borda Count, named after Jean-Charles Borda (1781). The procedure may be explained as follows. Suppose, there are $\mathrm{m}$ actions to choose from. Give each of these $\mathrm{m}$ actions a score from 1 to $\mathrm{m}$ on its ranking in a voter's preference ordering. The highest ranking action receives $m$, next $m-1$ points and so on. The action with highest points in the aggregate, is the winner. In case of a tie, an adjusted Borda rule may be used (see Black, 1958). Young (1974) contains a discussion on the axiomatic basis for the Borda rule. One difficulty with the Borda Count is that it may not be strategy-proof (Sen, 1984). An individual in the group may be able to manipulate the group decision by deliberately misrepresenting his preferences if he knows others' preferences. However, if the group is large, it is difficult to know everybody else's preference ordering and it is also unlikely that a single in-

* The paper was written when the author was visiting the Center of Economic Studies at Munich University, Germany. He would like to thank S. Vicary, H.W. Sinn, A. Razin and, particularly, the referee of this journal for helpful suggestions. The responsibility for any error lies with the author.

Originally published in Public Choice (1994) 80: 23-34. 
dividual can manipulate the group decision (Mueller, 1989). Another problem with Borda Count is that it assumes the intensity of the preferences to be equally spaced by ranking preferences by successive integers which is a special case of linear scoring rule. In this paper, we shall examine how to eliminate some actions without using any scoring rule and discuss methods of selecting the best action which may be regarded as nonlinear extensions of the Borda rule.

\section{The Borda procedure re-examined}

Consider a finite set of actions $X=\left(x_{1}, \ldots, x_{m}\right)$ from which a particular action has to be selected by a group of $\mathrm{N}$ individuals. Following the Borda rule, each individual assigns $m$ points to the most preferred action, $m-1$ points to second best action and soforth. Consider the proportion of individuals who regard $x_{i}$ as the best action, the proportion of individuals who regard $x_{i}$ as the second best action and so on. $f_{i}\left(x_{i}\right)$ and $f_{j}\left(x_{j}\right)$ represent the distribution of these proportions for $x_{i}$ and $x_{j}$ respectively. For example, $f_{j}\left(x_{j}=3\right)$ is the number of persons who considers $x_{j}$ as their third choice, from the bottom of their preference list, divided by size of the group $(N)$. Clearly, $\underset{x_{j}=1}{m} f_{j}\left(x_{j}\right)=1$. Borda rule implies the selection of that action which has the highest expected count, i.e., $x_{i}$ will be selected over $x_{j}$ if $\sum_{x_{i}=1}^{m} x_{i} f_{i}\left(x_{i}\right)$ is greater than $\underset{x_{j}=1}{m} x_{j} f_{j}\left(x_{j}\right) ; x_{i}$, $\mathrm{x}_{\mathrm{j}}=1,2, \ldots, \mathrm{m}$. Suppose, there are three alternatives and the first action $\left(\mathrm{x}_{1}\right)$ is given 1 point by $20 \%$ of the voters, 2 points by $50 \%$ of the voters and 3 points by $30 \%$ of the voters. If an individual is selected arbitrarily from the group, the expected score of $x_{1}$ is $1(.2)+2(.5)+3(.3)=2.1$. One way to justify the Borda procedure is to argue that if a person is selected arbitrarily from the group, the action which is likely to receive the highest expected score should win. This argument may also be used to justify the majority rule. Consider the case of two alternatives, one of which receives $55 \%$ of the votes. Why should the losers accept the verdict of the majority? An answer may be the following. In a democratic society, there should be no discrimination amongst voters. If an individual is selected arbitrarily as the representative voter, the action which wins the majority vote is more likely to be selected by this representative individual. The annonymity (equal weight) condition may thus be used to justify the majority rule. It must be clearly understood that the outcome of the simple Borda procedure depends not only on the individual preferences but also on the linear scoring system accepted by the group as a whole. The linear scoring system relates the score and the rank (place in individual preference ordering) in the following way: 


$$
\mathrm{S}(\mathrm{r})=\alpha+\beta . \mathrm{r}, \beta>0 .
$$

In Eq.(1), $\mathrm{r}$ represents the rank. In simple Borda rule, $\alpha=0$ and $\beta=1$. So long the scoring system is linear, variation in $\alpha$ and $\beta$ does not affect the choice by Borda rule.

Once the Borda procedure is viewed in the way as outlined above, shortcomings of the simple Borda rule becomes quite obvious. It is apparent that in selecting the best action, the entire profile of each distribution $f_{k}\left(x_{k}\right), x_{k} \in X$ should be compared rather than the expected values. Again, consider the case of 4 alternatives: $x_{1}, x_{2}, x_{3}, x_{4}$. Suppose, $x_{4}$ receives 4 points from $70 \%$ of the voters, $x_{1}$ receives 4 points from $30 \%$ of the voters and all voters give 3 points to $x_{3}$ and 2 points to $x_{2}$. The expected score of $x_{4}$ is $3.1 v i s-\grave{a}$-vis the expected score of 3 for $x_{3}$. If $x_{4}$ is chosen, $30 \%$ of the voters will receive the worst deal. There is a trade off between gain in expected score and the intensity of dislike from the losers. If the group is sensitive about the selection of an action which is ranked significantly low in the preference ordering of a substantial minority in the group, it may use the following nonlinear value function known as the adjusted mean criterion (also see Chew, 1983):

$$
\mathrm{V}(\mathrm{i})=\frac{\sum_{\mathrm{x}_{1}=1}^{\mathrm{m}} \mathrm{x}_{\mathrm{i}} \cdot \mathrm{f}_{\mathrm{i}}\left(\mathrm{x}_{\mathrm{i}}\right)}{\left[\sum_{\mathrm{x}_{\mathrm{i}}=1}^{\mathrm{m}} \tau\left(\mathrm{x}_{\mathrm{i}}\right) \cdot \mathrm{f}_{\mathrm{i}}\left(\mathrm{x}_{\mathrm{i}}\right)\right]^{\beta}},
$$

where $\tau\left(\mathrm{x}_{\mathrm{i}}\right)$ is decreasing in $\mathrm{x}_{\mathrm{i}}$ and $\beta>0$. If larger proportion of individuals put $x_{i}$ at the bottom of their preference ordering relative to $x_{j}$, then under the value function $V$ the Borda index is downwardly adjusted for $x_{i}$. $V(i)$ is a nonlinear value function because it is a nonlinear mapping of the function $\mathrm{f}_{\mathrm{i}}\left(\mathrm{x}_{\mathrm{i}}\right)$ on the real line. Note, if $\tau\left(x_{i}\right)$ is chosen as $\left(x_{i}-\bar{x}_{i}\right)^{2}$ with $\beta=\frac{1}{2}$, then Eq.(2) is the inverse of the coefficient of variation. Note, $\bar{x}_{i}=\Sigma x_{i} \cdot f_{i}$ for all $i$. Higher expected score increases the attraction of an alternative and higher variance of $\mathrm{f}_{\mathrm{i}}\left(\mathrm{x}_{\mathrm{i}}\right)$ reduces the attraction. The reader may note a conceptual affinity between risk aversion and a bias towards the voters who are not doing well under the Borda rule. The former implies a bias against loss making, the later implies a bias against imposing a bad choice on some members of the group.

The Borda rule uses the information contained in the ranking of actions by each individual according to his preference. However, even if one action is preferred to another by two individuals, the intensity of preferences may vary. However, it is not possible to make interpersonal comparison of the intensity of preferences and, under the Borda rule, the group collectively treats each in-

Originally published in Public Choice (1994) 80: 23-34. 
dividual ranking of alternatives without any bias. However, without being biased towards any individual, the group may feel that pulling up some people at the bottom of their preferences by one mark at the cost of pulling down an equal number of people at the top of their preference ranking by one mark is justified. In such a case, the group may be motivated to use a value function like the adjusted mean criterion. The problem with the adjusted mean criterion as formulated in Eq.(2) is that it may lead to the selection of an inefficient action or alternative. Therefore, the application of such a criterion must be restricted to the set of efficient actions. In the next section, we shall explain the criterion of efficiency and its implications.

\section{The efficiency set}

One of the problems with the simple Borda procedure is that it adopts a linear scoring system. To say the least, ordering preference by successive integers is a crude method of ranking. To quote Black (1958: 65): “Our criticism is that no merit ranking of this kind exists; and if this is so, no possible justification for the Borda criterion can be got along these lines." Therefore, it is useful to see whether it is possible to eliminate certain actions from group choice without taking recourse to any kind of scoring system linear or nonlinear.

$$
\text { Let us define } F_{i}\left(x_{i}\right)=\sum_{x_{i}=1}^{t} f_{i}\left(x_{i}\right), 1 \leq t \leq m-1 \text {. }
$$

Definition 1. The action $\mathrm{x}_{\mathrm{i}}$ dominates the action $\mathrm{x}_{\mathrm{j}}$ in pairwise comparison if and only if $\mathrm{F}_{\mathrm{t}}\left(\mathrm{x}_{\mathrm{i}}\right)<\mathrm{F}_{\mathrm{t}}\left(\mathrm{x}_{\mathrm{j}}\right)$ for all $1 \leq \mathrm{t} \leq \mathrm{m}-1$. An action which is not dominated by any pairwise comparison is called a dominant action. The set of dominant actions is called the Efficiency Set.

The idea behind dominance is as follows. Suppose, each member of the group is given $k$ number of "chips" (interpret $k=m-t$ ) and is asked to assign one chip to each of $k$ actions at the top of their list of preferences. If action $x_{i}$ receives more chips than the action $x_{j}$ for all values of $k$, then the action $x_{i}$ dominates the action $x_{j}$ and the action $x_{j}$ should not be chosen by the group when $x_{i}$ is available. To put it in another way, suppose we ask the members of the group to select $k$ actions $(1 \leq k \leq m-1)$ from the top of their list of preferences. Let $x_{i}$ be an action which is chosen by a larger number of members than those choosing $x_{j}$. If $x_{i}$ is chosen by a larger number of members for all values of $\mathrm{k}$, then $\mathrm{x}_{\mathrm{i}}$ is said to dominate $\mathrm{x}_{\mathrm{j}}$. 
Proposition 1. The relation of dominance is transitive.

Proof: Suppose $\mathrm{x}_{\mathrm{i}}$ dominates $\mathrm{x}_{\mathrm{j}}$ and $\mathrm{x}_{\mathrm{j}}$ dominates $\mathrm{x}_{\mathrm{k}}$. Then by definition $\mathrm{F}_{\mathrm{t}}\left(\mathrm{x}_{\mathrm{i}}\right)$ $<F_{t}\left(x_{j}\right)$ and $F_{t}\left(x_{j}\right)<F_{t}\left(x_{k}\right)$ for all $1 \leq t \leq m-1$. Therefore, $F_{t}\left(x_{i}\right)<F_{t}\left(x_{k}\right)$ for all $t$, implying that $x_{i}$ must dominate $x_{k}$.

QED

Proposition 2. (Consistency) Consider two groups of individuals $\mathrm{N}_{1}$ and $\mathrm{N}_{2}$. If in both the groups $x_{i}$ dominates $x_{j}$ then also in the combined group $N=N_{1}$ $\cup \mathrm{N}_{2}, \mathrm{x}_{\mathrm{i}}$ dominates $\mathrm{x}_{\mathrm{j}}$.

Proof: Since in both the groups $(1,2), \mathrm{x}_{\mathrm{i}}$ dominates $\mathrm{x}_{\mathrm{j}}$.

$$
F_{t}^{k}\left(x_{j}\right)<F_{t}^{k}\left(x_{j}\right), k=1,2 \text {, for all } t<m-1 \text {. }
$$

Let $m_{k}=N_{k} /\left(N_{1}+N_{2}\right)$. The the following inequality holds,

$$
\sum_{\mathrm{k}} \mathrm{m}_{\mathrm{k}} \cdot \mathrm{F}_{\mathrm{t}}^{\mathrm{k}}\left(\mathrm{x}_{\mathrm{i}}\right)<\sum_{\mathrm{k}} \mathrm{m}_{\mathrm{k}} \cdot \mathrm{F}_{\mathrm{t}}^{\mathrm{k}}\left(\mathrm{x}_{\mathrm{j}}\right) \text { for all } \mathrm{t}<\mathrm{m}-1 \text {. }
$$

Noting that for the combined group $F_{t}\left(x_{i}\right)=\sum_{k} m_{k} \cdot F_{t}^{k}\left(x_{i}\right)$, the proposition follows immediately.

QED

Proposition 3. If $\mathrm{x}_{\mathrm{i}}$ dominates $\mathrm{x}_{\mathrm{j}}$, the total score of $\mathrm{x}_{\mathrm{i}}$ will be greater than that of $x_{j}$ whatever be the form of the scoring function provided the scores are increasing with the rank.

Proof: Total score of $\mathrm{x}_{\mathrm{i}}$ is given by $\mathrm{N} \Sigma \mathrm{x}_{\mathrm{i}} \cdot \mathrm{f}\left(\mathrm{x}_{\mathrm{i}}\right)$. Therefore, if the expected score of $x_{i}$ is greater than that of $x_{j}$ then it also holds for the total score. Hadar and Russell (1969, Theorems 1 and 2 ) contains the proof that the expected score of $x_{i}$ is greater than that of $x_{j}$ for any scoring function increasing with the rank, if and only if $x_{i}$ dominates $x_{j}$. In order to economize on space we refrain from reproducing the proof here.

QED

Proposition 4. For a finite group of individuals choosing from a finite set of actions, the Efficiency Set always exists.

Proof: Suppose the efficiency set is empty. If the efficiency set does not exist, then each action is dominated by at least one other action, e.g., $x_{i}$ is dominated by $x_{j}, x_{j}$ is dominated by $x_{k}$ and so on. By Proposition 2 , the relation of dominance is transitive and therefore non-cyclical. Consequently, the chain of dominance must lead to an action in the limit which dominates all other actions and is therefore undominated. This contradicts the assumption that the efficiency set is empty.

QED

Originally published in Public Choice (1994) 80: 23-34. 
Proposition 5. If an action exists which could win under the majority rule, then this action is included in the Efficiency Set.

Proof: If more than $\mathrm{N} / 2$ individuals prefer $\mathrm{x}_{\mathrm{i}}$ to other actions, $\mathrm{x}_{\mathrm{i}}$ is chosen under the majority rule. In this case $F_{t}\left(x_{i}\right)<F_{t}\left(x_{j}\right)$ for $t=m-1$ and for all $\mathrm{j} \neq \mathrm{i}$. Therefore, $\mathrm{x}_{\mathrm{i}}$ is an undominated action.

QED

Proposition 6. The action chosen by the Borda rule is contained in the efficiency set.

Proof: Let $\mathrm{x}_{\mathrm{i}}$ be the winner by the Borda rule and $\mathrm{x}_{\mathrm{j}}$ be another action which dominates $x_{i}$. By Proposition 4, under any scoring rule, the total score of $x_{j}$ must be greater than that of $x_{i}$. But we know that the winner by the Borda rule has the highest score under the linear scoring system. Therefore, $x_{j}$ can not dominate $\mathrm{x}_{\mathrm{i}}$.

QED

A group decision making process is said to be faithful (Young, 1974; Fishburn, 1979), if "the most preferred action(s) by the group" and "the most preferred action(s) by the individual" are same if the group is reduced to a single individual. The efficiency set satisfies this critierion of faithfulness.

Proposition 7. If the group consists of a single individual, the efficiency set picks up the action which is most preferred by the individual.

Proof: Suppose the group consists of a single individual and without any loss of generality assume that he prefers $x_{1}$ to all other actions. Then we have $F_{1}\left(x_{1}\right)=F_{2}\left(x_{1}\right)=\ldots \ldots=F_{m-1}\left(x_{1}\right)=0$. For $j \neq 1, F_{t}\left(x_{j}\right)$ must equal 1 for $t \geq t^{*}$ for some $t^{*} \leq m-1$. Therefore, $x_{1}$ must dominate all other actions and is the only element in the efficiency set.

QED

In general the efficiency set contains more than one action. In choosing between actions without selecting any specific scoring function (ordinal ranking) this is the limit of success. If the efficiency set contains a single element, then the Borda rule will pick it up. However, we do not know a priori that this is the case. The problem is, how to select an action from the efficiency set. The choice of the scoring system and the considerations regarding the method for processing the scores (value function) enters at this stage. The group can use a linear scoring system together with a non-linear value function like Eq.(2). But considerations for efficiency and dominance should have priority before the use of any scoring system, otherwise the use of a non-linear value function may result in the choice of a dominated action. 


\section{An alternative secondary criterion}

Eq.(2) offers us a secondary criterion which may be applied to choose from the efficiency set. In most cases of democratic decisions, the voting procedure pays special consideration to those who are not doing well under the voting mechanism. An extreme case is the provision of veto, which entitles a member to prevent the group from taking a decision which is not liked by the member. In this section, we shall propose an alternative secondary criterion which may be used to choose an action from the efficiency set. Suppose, the group uses a certain rank from the bottom in the preference list as the benchmark between bad ranks and acceptable ranks. For example, consider rank 2 as the benchmark rank. This is purely a reflection of the value judgments within the group. Let $\alpha_{j}(2)$ be the proportion of the population which ranks $x_{j}, j=1,2, \ldots, m$, at 2 or less. If $F_{j}(t)$ denotes the proportion of population which gives the action $\mathrm{x}_{\mathrm{j}}$ a rank of $\mathrm{t}$ or less, $\alpha_{\mathrm{j}}(2)=\mathrm{F}_{\mathrm{j}}(2)$. All the actions may be ranked according to $\alpha_{\mathrm{j}}$ and the group may decide to choose that action which minimizes $\alpha_{\mathrm{j}}(2)$. In case of a tie, the group may choose any one of them. We shall refer to this as the $\alpha(t)$-criterion where $t$ refers to the benchmark rank. By itself, $\alpha(t)-$ criterion is not a very attractive criterion but it can be used to arrive at a compromised solution.

Proposition 8. If $\mathrm{x}_{\mathrm{i}}$ is a winner under the $\alpha(\mathrm{t})$-criterion with no ties, for all values of $t \leq m-1$, then $x_{i}$ dominates all other actions and is a winner under the Borda rule.

Proof: If $\mathrm{x}_{\mathrm{i}}$ is a winner under the $\alpha(\mathrm{t})$-criterion with no ties for all $\mathrm{t}$, the $\mathrm{F}_{\mathrm{i}}(\mathrm{t})$ $<\mathrm{F}_{\mathrm{j}}(\mathrm{t})$ for all $\mathrm{t} \leq \mathrm{m}-1$ and $\mathrm{j} \neq \mathrm{i}$. Hence, $\mathrm{x}_{\mathrm{i}}$ dominates all $\mathrm{x}_{\mathrm{j}}, \mathrm{j} \neq \mathrm{i}$. Since $\mathrm{x}_{\mathrm{i}}$ dominates all other actions, it must be the only element in the efficiency set. Since the winner under the Borda rule is always contained in the efficiency set (Proposition 7 ), $x_{i}$ must be a winner under the Borda rule.

QED

Proposition 9. If $\mathrm{x}_{\mathrm{i}}$ is a winner under the $\alpha(\mathrm{t})$-criterion for any particular value of $t$, then $x_{i}$ is contained in the Efficiency Set.

Proof: If $\mathrm{x}_{\mathrm{i}}$ is a winner under the $\alpha(\mathrm{t})$-criterion for $\mathrm{t}=\mathrm{t}^{*}$, then $\mathrm{F}_{\mathrm{i}}\left(\mathrm{t}^{*}\right) \leq \mathrm{F}_{\mathrm{j}}\left(\mathrm{t}^{*}\right)$, $j \neq i$. By the definition of dominance, there is no $x_{j}$ which can dominate $x_{i}$. Therefore, $x_{i}$ must be included in the efficiency set.

QED

The $\alpha(t)$-criterion may, by itself, be viewed as an extreme criterion where the interest of the average member is totally dominated by the adversely affected group which may be very small. However, it is also true that the voting procedure which does not pay any attention to the size of the adversely affected

Originally published in Public Choice (1994) 80: 23-34. 
group may call the stability of the group in question. It seems natural that the group should consider both the expected rank of each action (derived from the Borda procedure) as well as its rank according to the $\alpha(\mathrm{t})$-criterion for an agreed value of $t=t^{*}$. This alternative secondary criterion may be expressed by a value function:

$$
\mathrm{v}(\mathrm{i})=\mathrm{v}\left(\mathrm{E}_{\mathrm{i}}, \alpha_{\mathrm{i}}\left(\mathrm{t}^{*}\right)\right), \mathrm{v}_{1}>0, \mathrm{v}_{2}<0
$$

The actions included in the efficiency set should be ranked according to the value function v(i) and the action with the highest value should be chosen by the group. The motivation behind such a value function is somewhat different from that of the adjusted mean criteria. In order to explain it, let us refer back to the example in Section 2, where choice has to be made from 4 alternatives. $70 \%$ of voters gives a mark of 4 to $x_{4}$ and $30 \%$ does the same to $x_{1}$. Everyone gives a mark of 2 to $x_{2}$ and 3 to $x_{3}$. Suppose the group hates the idea to force its members in accepting the worst outcome and sets its benchmark at the modest level of $t=1$. The group selects that alternative which minimizes the proportion of population for which it is the worst outcome. The alternatives $\mathrm{x}_{1}$ and $\mathrm{x}_{4}$ drops out of consideration with $\alpha_{2}(2)=\alpha_{3}(2)=100 \%$. However, the expected marks or the Borda counts for $x_{2}$ and $x_{3}$ are 2 and 3 respectively. Given a value function like Eq.(3), the alternative $x_{3}$ is chosen by the group although $\mathrm{x}_{4}$ has a higher Borda count of 3.1.

The adjusted mean criterion is essentially a generalized Borda procedure with a sympathetic consideration for those members of the group who are badly affected by the group choice. In Eq. (2) if $\tau\left(x_{i}\right)=1$ for all $i$ (or $\beta$ is close to 0 ), the Borda rule is optimal. Any deviation from the Borda rule will essentially depend on the relative magnitude of weights, $\tau\left(\mathrm{x}_{\mathrm{i}}\right)$ assigned by the group to its members having a raw deal and to the extent $(\beta)$ by which the winners in the group are prepared to accomodate the losers. It should be emphasized that like Eq.(2), Eq.(3) only provides us with a secondary criterion to be applied only to the set of efficient actions, otherwise it may pick up an inefficient action which is certainly not desirable.

\section{The consistency axiom and quasi-linearity}

One feature of the Borda rule is that it satisfies the consistency assumption (Young, 1974); Fishburn, 1979). Stating the axiom in a simple manner, if using a choice procedure two groups of voters $N_{1}$ and $N_{2}$ choose $x_{j}$ separately then the choice procedure is said to be consistent if the combined group is also required to choose $x_{j}$. Furthermore, any alternative not chosen by any one of the groups is, in some sense, not as "good" as an alternative chosen by both. This

Originally published in Public Choice (1994) 80: 23-34. 
concept of consistency does not extend to efficiency sets or to the alternative secondary rules discussed in this paper. The reason why they do not satisfy the consistency axiom requires an understanding of the nature of restrictions imposed on the choice procedure by the consistency axiom.

Let $f=\left\{f_{1}\left(x_{1}\right), f_{2}\left(x_{2}\right), \ldots \ldots, f_{m}\left(x_{m}\right)\right\}$ be the set of score distributions and $F$ be the set of all possible $f, F=[f\}$. The choice function is a mapping $\Phi$ which maps $\mathrm{F}$ on $\mathrm{X}$.

$$
\Phi: F \rightarrow X
$$

Definition 2. The mapping $\Phi$ is said to be quasi-linear if, $\Phi\left(\mathrm{f}^{*}\right) \cap \Phi\left(\mathrm{f}^{* *}\right)=$ $\left\{x_{j}\right\}$ implies that $\Phi\left(\alpha f^{*}+(1-\alpha) f^{* *}\right) \supset\left\{x_{j}\right\}$. where $0 \leq \alpha \leq 1$.

Consider two groups of voters $\mathrm{N}_{1}$ and $\mathrm{N}_{2}$ with two profiles, $\mathrm{f}^{*}$ and $\mathrm{f}^{* *}$, of the score distributions of the available actions for the two groups. For the combined group, let $f_{a}$ be the profile of score distributions of the available actions. It is clear that $\mathrm{f}_{\mathrm{a}}=\alpha \mathrm{f}^{*}+(1-\alpha) \mathrm{f}^{* *}$ where $\alpha=\mathrm{N}_{1} /\left(\mathrm{N}_{1}+\mathrm{N}_{2}\right)$. Remember, $\mathrm{f}^{*}$ and $\mathrm{f}^{* *}$ are vectors of density functions. Suppose, $\Phi\left(\mathrm{f}^{*}\right)=\Phi\left(\mathrm{f}^{* *}\right)=\mathrm{x}_{\mathrm{j}}$ then quasi-linearity implies that $\Phi\left(\mathrm{f}_{\mathrm{a}}\right)=\mathrm{x}_{\mathrm{j}}$.

Definition 3. The mapping $\Phi$ is consistent if for $\Phi\left(\mathrm{f}^{*}\right) \cap \Phi\left(\mathrm{f}^{* *}\right) \neq \emptyset, \Phi\left(\mathrm{f}^{*}\right) \cap$ $\Phi\left(f^{* *}\right) \subset \Phi\left(f_{a}\right)$.

Since $\mathrm{f}_{\mathrm{a}}=\alpha \mathrm{f}^{*}+(1-\alpha) \mathrm{f}^{* *}$ with $0 \leq \alpha \leq 1$, consistency implies quasilinearity. Apparently, this is a severe restriction on the choice function $\Phi$ and there is no a priori reason why $\Phi$ should satisfy this restriction. The Borda rule satifies this restriction in a trivial way. Since under the Borda rule $\Phi$ selects $x_{j}$ to maximise $E\left(f_{j}\left(x_{j}\right)\right)$, it is clear that when both groups choose $x_{j}^{*}$, for the combined group

$$
\underset{x_{i}}{\operatorname{Max}} E\left(f_{a}\right)=\underset{x_{i}}{\operatorname{Max}}\left[\alpha E\left(f^{*}\right)+(1-\alpha) E\left(f^{* *}\right)\right]=E\left(f_{a, j^{*}}\left(x_{j}^{*}\right)\right)
$$

because $f_{a}$ is a convex combination of $f^{*}$ and $f^{* *}$. Therefore $x_{j}^{*}$ is also in the choice set of the combined group. The density function of $x_{j}^{*}$ is denoted above by $f_{a, j^{*}}\left(x_{j^{*}}\right)$. The consistency property need not hold good when the group decision depends not only on the expected value but also on other features of the score density functions. Suppose, the decision of a group depends both on the expected score and the second order non-central moment $\left(\mu_{2}\right)$ of the score density functions and the choice is made using a value function like Eq.(2). This is done by choosing $\tau\left(x_{i}\right)=x_{i}^{2}$, for all $i$. In this case, by Eq.(2), 


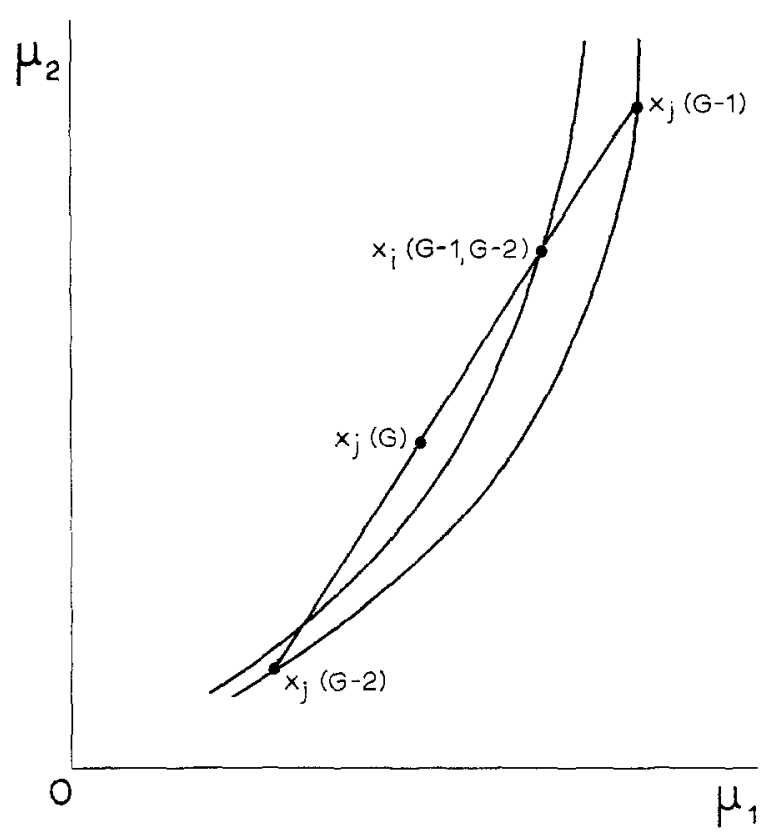

Figure 1.

$$
\mathrm{V}(\mathrm{i})=\mu_{1} /\left(\mu_{2}\right)^{\beta}, \beta, 0 .
$$

For the combined group, the score density function of the $i$-th action is: $f_{a}\left(x_{i}\right)$ $=\alpha \mathrm{f}_{1}\left(\mathrm{x}_{\mathrm{j}}\right)+(1-\alpha) \mathrm{f}_{2}\left(\mathrm{x}_{\mathrm{i}}\right)$ where $\alpha=\mathrm{N}_{1} /\left(\mathrm{N}_{1}+\mathrm{N}_{2}\right)$. Therefore, the $\mathrm{k}$-th moment of the score distribution of the combined group is:

$$
\mu_{\mathrm{k}}^{\mathrm{a}}(\mathrm{i})=\Sigma \mathrm{f}_{\mathrm{a}}\left(\mathrm{x}_{\mathrm{j}}\right) \cdot \mathrm{x}_{\mathrm{i}}^{\mathrm{k}}=\alpha \cdot \mu_{\mathrm{k}}^{1}(\mathrm{i})+(1-\alpha) \cdot \mu_{\mathrm{k}}^{2}(\mathrm{i})
$$

In other words, the $\mathrm{k}$-th order moment of $\mathrm{x}_{\mathrm{i}}$ for the combined group is a convex combination of the k-th order moment of $x_{i}$ for each group separately. For the combined group, the expected score $\left(\mu_{1}\right)$ and $\mu_{2}$ for the i-th action is: $\mathrm{E}_{\mathrm{i}}(\mathrm{a})=\alpha \mathrm{E}_{\mathrm{i}}(1)+(1-\alpha) \mathrm{E}_{\mathrm{i}}(2)=\mu_{1, \mathrm{i}}(\mathrm{a})$ and $\mu_{2, \mathrm{i}}(\mathrm{a})=\alpha \mu_{2, \mathrm{i}}(1)+(1-\alpha) \mu_{2, \mathrm{i}}(2)$. In other words, for a given action $i$, the values of $\mu_{1}$ and $\mu_{2}$ for the combined group would be the convex combination of the values in each group separately.

In Figure 1, the values of $\left(\mu_{1}, \mu_{2}\right)$ for the action $\mathbf{j}$ has been presented as $x_{j}(G-1)$ for group 1 and as $x_{j}(G-2)$ for group 2. For the combined group (G), $\left(\mu_{1}, \mu_{2}\right)$ is given by $x_{j}(G)$. If in Eq.(5) we assume $\beta=1$, then the group indifference curves in the $\left(\mu_{1}, \mu_{2}\right)$ plane are given by equations

$$
\mu_{1}=\mathrm{c} \mu_{2}, \mathrm{c}>0
$$


and are representable by straight lines originating from the origin in Figure 1. In such a case, the consistency property for group decisions will hold good. But there is no reason why $\beta$ should be equal to 1 . For $\beta<1$, the indifference curves in the $\left(\mu_{1}, \mu_{2}\right)$ plane are given by

$$
\mu_{1}=\mathrm{c} \mu_{2}{ }^{\beta}, \beta<1, \mathrm{c}>0
$$

Such indifference curves have been shown in Figure 1. Suppose, the score distribution for the $\mathrm{i}$-th action has the same values for $\mu_{1}$ and $\mu_{2}$ for both the groups and is denoted by $x_{i}(G-1, G-2)$ in Figure 1 . Both groups prefer the $\mathrm{j}$-th action to the $\mathrm{i}$-th action. But given appropriate relative sizes, the $\mathrm{i}$-th action is preferred to the $\mathrm{j}$-th action by the combined group, i.e., $x_{i}(G-1, G-2)$ is preferred to $x_{j}(G)$. Examples of such violations of the consistency property may be easily constructed. The only situation where such violations may not occur is when $\beta=1$. Then the preferences are quasi-linear, i.e., the indifference curves in Figure 1 are straight lines and in this case rays through the origin. Further, it may be pointed out that in the context of our example, straight line indifference curves have a counter-intuitive implication. Let $\mathrm{V}$ denote the variance in score. We know that by definition $V=\mu_{2}-\mu_{1}^{2}$. In the case of straight line indifference curves along any particular indifference curve we have $\mu_{2}=\mathrm{k}+\lambda \mu_{1}, \mathrm{k}, \lambda>0$. From these two relationships, we obtain $\mathrm{V}=$ $\mathrm{k}+\lambda \mu_{1}-\mu_{1}^{2}$. Therefore, along any indifference curve $\mathrm{dV} / \mathrm{d} \mu_{1}=\lambda-2 \mu_{1}$. For a sufficiently high $\mu_{1}, \mathrm{dV} / \mathrm{d} \mu_{1}<0$. In other words, if two actions $\mathrm{x}_{\mathrm{i}}$ and $\mathrm{x}_{\mathrm{j}}$ have same expected score $\left(\mu_{1}\right)$ but scores are more polarized with respect to $x_{j}$, the group prefers $x_{j}$ to $x_{i}$. On the other hand, using Eq.(8) we get, $V=$ $\left[(1 / \mathrm{c}) \mu_{1}\right]^{1 / \beta}-\mu_{1}^{2}$. It is easy to see that for $0<\mathrm{c}<1$ and $\beta<1 / 2, \mathrm{dV} / \mathrm{d} \mu_{1}$ $>0$ everywhere along any indifference curve.

One can also easily check that the combined group index for $\alpha_{i}\left(t^{*}\right)$ is the convex combination of separate group indices for $\alpha_{\mathrm{i}}\left(\mathrm{t}^{*}\right)$. Thus the consistency condition is satisfied when the value function v in Eq.(3) is linear. In Eq.(2), consistency requires that the preferences are quasi-linear. If preferences are nonlinear, as one expects them to be, the choice procedures are likely to violate the consistency axiom. Also note, the value function creeps into our discussion because, unlike Borda, we are considering the case where the choice procedure takes into account more than one property of the score distribution functions.

\section{Conclusion}

Any problem of choice has two aspects: (a) consideration of efficiency leading to the identification of an efficiency set; (b) selection of a criterion to choose from the efficiency set. In welfare economics, the Pareto-criterion determines

Originally published in Public Choice (1994) 80: 23-34. 
the efficiency set. Then we require a social welfare function or a criterion of fairness to select from the Pareto-efficient distributions. In problems of decision making under uncertainty a similar method is called for (Fishburn, 1964). In financial economics, consideration of efficient portfolios precedes the selection of the optimal portfolio. Similary, in problems of group decisions which involve processing of individual preferences, consideration of efficiency takes precedence over value judgments. We have discussed the definition and the nature of the efficiency set in the context of group decision making. The value judgments of a group are reflected in their choice of a value function. We have discussed two secondary criteria, namely adjusted mean criterion and the $\alpha(\mathrm{t})$ criterion, which pay some attention to the size of members badly affected by the choice. The consistency axiom of group choice is likely to be violated under such circumstances.

\section{References}

Black, D. (1958). The theory of committees and elections. Cambridge (U.K.): Cambridge University Press.

Borda, J.C. de. (1781). Mémorie sur les Elections au Scrutin. Histoire de l'Academie Royale des Sciences.

Chew, S.H. (1983). A generalization of the quasi-linear mean with applications to the measurement of income inequality and decision theory resolving the Allais Paradox. Econometrica 51(4): $1065-1092$.

Fishburn, P.C. (1964). Decision and value theory. New York: Wiley.

Fishburn, P.C. (1979). Symmetric and consistent aggregation. In J.-J. Laffont (Ed.), Aggregation and revealation of preferences: Symmetric and consistent aggregation in aggregation and revealation of preferences, 201-218. Amsterdam: North-Holland.

Hadar, J. and Russell, W.R. (1969). Rules for ordering uncertain prospects. American Economic Review 59(1): 25-34.

Mueller, D. (1989). Public Choice II. Cambridge (U.K.): Cambridge University Press.

Sen, A.K. (1970). Collective choice and social welfare. London: Holden-Day Inc.

Sen, M. (1984). Strategy-proofness of a class of Borda rules. Public Choice 43(3): 251-285.

Young, H.P. (1974). An axiomatization of Borda's rule, Journal of Economic Theory 9(1): $43-52$. 